\title{
Threshold Dissociation and Molecular Modeling of Transition Metal Complexes of Flavonoids
}

\author{
Junmei Zhang and Jennifer S. Brodbelt \\ Department of Chemistry and Biochemistry, University of Texas, Austin, Texas, USA \\ Junmei Wang \\ Encysive Pharmaceuticals Inc., Houston, Texas, USA
}

\begin{abstract}
The relative threshold dissociation energies of a series of flavonoid/transition metal/auxiliary ligand complexes of the type $\left[\mathrm{M}^{\mathrm{II}} \text { (flavonoid }-\mathrm{H} \text { ) auxiliary ligand }\right]^{+}$formed by electrospray ionization (ESI) were measured by energy-variable collisionally activated dissociation (CAD) in a quadrupole ion trap (QIT). For each of the isomeric flavonoid diglycoside pairs, the rutinoside (with a 1-6 inter-saccharide linkage) requires a greater CAD energy and thus has a higher dissociation threshold than its neohesperidoside (with a 1-2 inter-saccharide linkage) isomer. Likewise, the threshold energies of complexes containing flavones are higher than those containing flavanones. The monoglycoside isomers also have characteristic threshold energies. The flavonoids that are glycosylated at the 3-O- position tend to have lower threshold energies than those glycosylated at the 7-O- or 4'-O- position, and those that are C- bonded have lower threshold energies than the $\mathrm{O}$ - bonded isomers. The structural features that substantially influence the threshold energies include the aglycon type (flavanone versus flavone), the type of disaccharide (rutinose versus neohesperidose), and the linkage type (Obonded versus C-bonded). Various computational means were applied to probe the structures and conformations of the complexes and to rationalize the differences in threshold energies of isomeric flavonoids. The most favorable coordination geometry of the complexes has a plane-angle of about $62^{\circ}$, which means that the deprotonated flavonoid and 2,2'-bipyridine within a complex do not reside on the same plane. Stable conformations of five cobalt complexes and five deprotonated flavonoids were identified. The conformations were combined with the point charges and helium accessible surface areas to explain qualitatively the differences in threshold energies for isomeric flavonoids. (J Am Soc Mass Spectrom 2005, 16, 139-151) ( 2004 American Society for Mass Spectrometry
\end{abstract}

$\mathrm{T}$ The antioxidant abilities and chemopreventive properties [1-3] of flavonoids have attracted great attention in recent years [4-19], especially because of the broad prevalence of flavonoids in many common fruits and vegetables. Structural characterization and isomer differentiation is a prerequisite for understanding the bioavailability, metabolism, pharmacokinetics, and structure/activity relationships of the flavonoids. However, structural characterization has been a particularly challenging analytical problem due to the large number and small structural differences of the flavonoids. Differentiation of the flavonoids is not easy because thousands of them share a common phenyl-benzopyrone skeleton (Figure 1), and they differ from one another only by the positions and numbers of hydroxyl or methoxylated functional groups, as well as

Published online December 9, 2004

Address reprint requests to Dr. J. S. Brodbelt, Department of Chemistry and Biochemistry, University of Texas, 1 University Station A5300, Austin, TX 78712-0165, USA. E-mail: jbrodbelt@mail.utexas.edu the inter-saccharide linkage, and the position and number of different saccharides involved in glycosylation [20]. Tandem mass spectrometry with soft ionization methods such as electrospray ionization (ESI) and fast atom bombardment (FAB) has been used with some success for structural characterization of flavonoids [4-19]. Both deprotonation [4-11] and protonation [7, 8, 12-16] modes have been used with collisionally activated dissociation (CAD) for analysis of flavonoids. Due to their acidic nature, flavonoids usually give higher ion abundances upon deprotonation in the negative ESI mode than via protonation in the positive ESI mode. Even greater ion abundances and more diagnostic fragmentation patterns of flavonoids have been reported based on a metal complexation mode in conjunction with CAD [10, 17-19]. Electrospray ionization of solutions containing a flavonoid, a transition metal salt, and an auxiliary ligand results in formation of complexes of the type $\left[\mathrm{M}^{\mathrm{II}}\right.$ (flavonoid $-\mathrm{H}$ ) auxiliary ligand] ${ }^{+}$where $\mathrm{M}^{\mathrm{II}}$ is the transition metal ion. These complexes have allowed successful differentiation of 


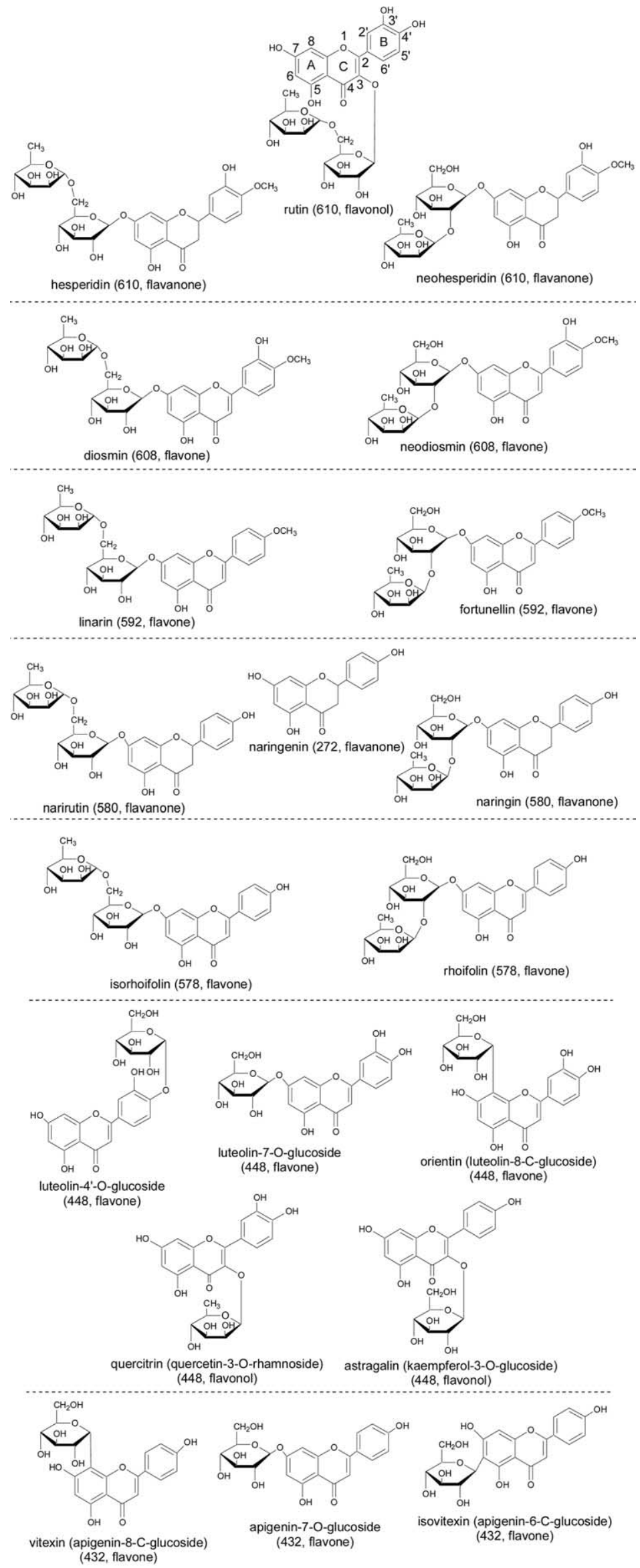

Figure 1. Flavonoid structures (molecular weight, class of flavonoid). 
flavonoid isomers [10,18, 19], and the CAD patterns can be "tuned" via changing the auxiliary ligand [19]. However, little is known about the specific structures of the metal complexes, yet they have greatly facilitated isomer differentiation and thus merit further fundamental investigation.

The early work of the McLuckey [21] and Brodbelt [22] groups proved the feasibility of using energy-variable $\mathrm{CAD}$ and threshold measurements in a quadrupole ion trap (QIT) to probe in a semi-quantitative way the relative binding energies of gas-phase ions. Several recent examples have used such a strategy to study the relative binding energies of pyridyl ligand/metal complexes [23], size effects on the stability of noncovalent complexes [24], the relative binding energies of nitrogen bases to metalloporphyrin cations [25], and factors impacting the strength of hydrogen-bonding interactions between polyethers and protonated amines [26] or peptides [27]. In the current study, energy-variable CAD and threshold measurements have been used to gain insight into the nature of the flavonoid/transition metal/auxiliary ligand complexes and to relate the differences in the threshold energies of isomeric complexes to specific structural features of the flavonoids, such as whether they are flavones versus flavanones, rutinosides versus neohesperidosides, glycosylated at the 3-versus 7- position, and C- bonded versus $\mathrm{O}-$ bonded glycosides. To rationalize the correlation between the structures of the flavonoid complexes and their threshold energies, various quantum mechanics $(\mathrm{QM})$ and molecular mechanics (MM) means have been used to optimize the structures and conformations of the cobalt complexes and deprotonated flavonoids. Point charges and helium accessible surface areas have been calculated to facilitate explanation of the experimental threshold results.

\section{Experimental}

\section{Chemical Reagents}

Hesperidin, naringenin, naringin, neohesperidin, and rutin (Figure 1) were purchased from Sigma (St. Louis, MO). Apigenin-7-O-glucoside, astragalin (kaempferol-3-Oglucoside), diosmin, isorhoifolin, isovitexin (apigenin6-C-glucoside), luteolin-4'-O-glucoside, luteolin-7-Oglucoside, narirutin, neodiosmin, orientin (luteolin8-C-glucoside), quercitrin (quercetin-3-O-rhamnoside), rhoifolin, and vitexin (apigenin-8-C-glucoside), (Figure 1) were purchased from Indofine (Somerville, NJ). Fortunellin and linarin (Figure 1) were purchased from Extrasynthese (Genay, France). 2,2'-Bipyridine (bipy), 4,7-dimethyl-1,10-phenanthroline (dmphen), 4,7-diphenyl-1,10-phenanthroline (dpphen), and 1,10-phenanthroline (phen) (Figure 2) as well as $\mathrm{CoBr}_{2}, \mathrm{NiBr}_{2}$, and $\mathrm{CuBr}_{2}$ were purchased from Aldrich (Milwaukee, WI). All the above compounds were used without further purification. All the stock $\left(10^{-4}-10^{-3} \mathrm{M}\right)$ solutions of flavonoids, metals $\left(10^{-3}-10^{-2} \mathrm{M}\right)$, and auxiliary ligands $\left(10^{-3}-10^{-2} \mathrm{M}\right)$ were prepared in HPLC grade methanol.
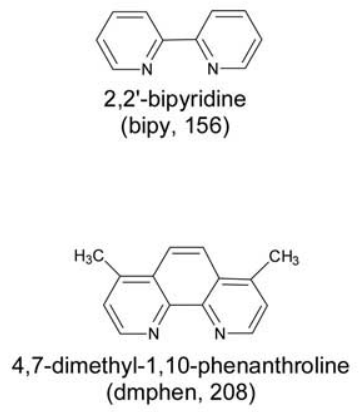

(dmphen, 208)
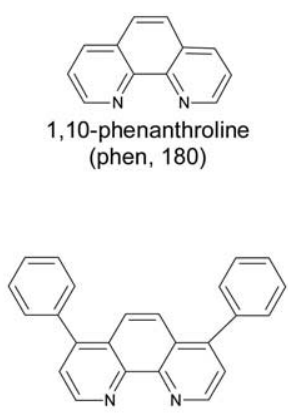

4,7-diphenyl-1,10-phenanthroline (dpphen, 332)

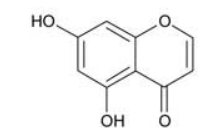

5,7-dihydroxy-4-chromone (178)

Figure 2. Structures of auxiliary ligands (abbreviation, molecular weight) and model compound 5,7-dihydroxy-4-chromone (molecular weight).

All the working solutions of flavonoid/transition metal/auxiliary ligand complexes (1:1:1, $1 \times 10^{-5} \mathrm{M}$ each) were also prepared in HPLC grade methanol.

\section{Threshold Dissociation}

A Thermo Finnigan LCQ Duo quadrupole ion trap (QIT) instrument equipped with an electrospray ionization (ESI) source (San Jose, CA) was used for all the energy-variable CAD experiments. The flow rate of a flavonoid/metal complex solution (1:1:1 flavonoid: transition metal: auxiliary ligand, $1 \times 10^{-5} \mathrm{M}$ each) was set at $5 \mu \mathrm{L} / \mathrm{min}$. The heated capillary temperature was kept at $200{ }^{\circ} \mathrm{C}$. The ESI spray voltage was $+4.5 \mathrm{kV}$ and the ionization time was $10 \mathrm{~ms}$. The other instrumental parameters were tuned to optimize the relative abundance of the $\left[\mathrm{Co}^{\mathrm{II}}\right.$ (neohesperidin - $\mathrm{H}$ ) bipy ${ }^{+}$complex. The above tune file was used for all the metal complexes. An isolation window of $2.0 \mathrm{~m} / \mathrm{z}$ was typically used. Following isolation, the complex was subject to collisional activation $\left(\mathrm{q}_{\mathrm{z}}=0.25\right.$, activation time $=30$ $\mathrm{ms}$ ). For the energy-variable CAD experiments, the normalized CAD energy ( $\%$ of $\left.5 \mathrm{~V}_{o-p}\right)$ was increased in $0.5 \%$ increments, and the relative abundances of the product ions as well as the parent ion were monitored. Each spectrum was an average of 100 scans. The isomers in each isomeric series were run back-to-back on the same day and repeated one to four times for reliable comparison.

When comparing the threshold energies of the metal complexes of glycosides and their aglycons or the complexes involving different auxiliary ligands, the threshold energies were corrected for the degrees of freedom (DOF) of the complexes relative to the $\left[\mathrm{Co}^{\mathrm{II}}\right.$ (flavonoid glycoside $-\mathrm{H}$ ) bipy $]^{+}$complexes. The correction was made using the following formula: thresh- 
old $($ corrected $)=$ threshold $($ exp. $) \times$ [no. of DOF $($ reference complex)]/[no. of DOF (complex of interest)] $[24,28,29]$. The usefulness of such a simple linear DOF correction as above has been demonstrated in the literature $[24,28,29]$.

\section{Molecular Modeling}

All the molecular modeling work was performed on the IBM pSeries 690 supercomputer of NCSA (www.ncsa. uiuc.edu).

Coordination geometry and structure optimization. The Gaussian 03 software package [30] was used for the ab initio calculations. The $\mathrm{A}$ and $\mathrm{C}$ rings of a deprotonated flavonoid and 2,2'-bipyridine define two planes. The most favorable plane-angle between the two planes was determined by a plane-angle scanning study (from 0 to $360^{\circ}$ with a step of $90^{\circ}$ ) at the B3LYP $/ 6-31 \mathrm{G}^{*}$ level for a model compound 5,7-dihydroxy-4-chromone. The structures of three flavonoid aglycon complexes, hesperetin, quercetin, and diosmetin were then optimized at the same level using the most favorable geometry obtained from the model compound. The deprotonation site of a flavonoid within its complexes was fixed at the $5-\mathrm{OH}$ position according to the coordination screening study reported by Satterfield et al. [17]. The structures of the same flavonoids without metal complexation (deprotonated flavonoids) were also optimized at the B3LYP/6-31G* level, and the most acidic site of a flavonoid was considered to be the deprotonation site, which was readily available for hesperetin and quercetin [31]. For diosmetin, the acidities of its hydroxyl groups were calculated at the same level [31].

Force field parameterization. Frequency analysis was performed on the model complex after the B3LYP/6$31 G^{*}$ optimization. The QM structural parameters as well as the vibrational frequencies were used as references to derive the missing force field parameters (bond lengths, bond angles, and torsional angles involving the metal cobalt) in MM studies. The missing MM force field parameters involving cobalt $\left(\mathrm{k}_{\mathrm{r}}, \mathrm{k}_{\theta}, \nu_{\mathrm{n}}, \varphi\right.$, etc.) were derived by varying the parameters such that the MM energy and frequencies reproduced the QM results. All the MM calculations including molecular dynamics (MD) simulations were performed with AMBER8 [32] using a general AMBER force field (GAFF) [33, 34].

Conformational searches. Both the cobalt complexes of five flavonoid diglycosides (hesperidin, neohesperidin, rutin, diosmin, and neodiosmin) and the deprotonated species were built by splicing the corresponding aglycon species (section 2.3.1) and the disaccharide portion

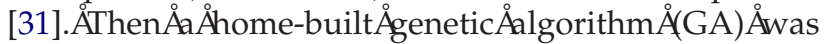
applied to explore the conformational space. For each cobalt complex or deprotonated flavonoid, 10 GA runs were performed to optimize the non-ring rotatable torsional angles and to lower the energies. A $10 \mathrm{~ns}$ gas phase MD simulation was then followed to further relax the best (having the lowest energy) GA structure. All the MD simulations were carried out at $298 \mathrm{~K}$ and 250 snapshots were collected after an initial equilibration of $2.5 \mathrm{~ns}$.

Helium accessible surface area calculations. A custombuilt program was used to calculate the helium accessible surface area, which is defined as the locus of the center of a helium sphere as it rolls over the van der Waals surface of a complex in study. The radius of the probe sphere, $1.4 \AA$ in this work, is the van der Waals radius of helium.

\section{Results and Discussion}

Our objective was to develop a better understanding of the factors that influence fragmentation of the metal complexes of flavonoids in the gas phase, thus allowing predictions of fragmentation behavior (fragmentation pathways and dissociation energetics) of new flavonoids or assisting in deciphering the structures of unknown flavonoids. Our strategy involved using energy-variable CAD, in which the applied collisional activation voltage is increased incrementally as the intensities of the parent ions and fragment ions are monitored, to determine the threshold energies of isomeric complexes and then to correlate this information with specific structural features of the flavonoids. Extensive molecular modeling was performed using various computational means to obtain the most stable structures and conformations of the cobalt complexes as well as the deprotonated flavonoids. The combination of conformations, point charges, and helium accessible surface areas was used to explain the experimental threshold data.

\section{Threshold Energies}

Seven isomeric series of flavonoid glycosides including eight monoglycosides and eleven diglycosides were used in this work. The representative CAD spectra of the $\left[\mathrm{Co}^{\mathrm{II}}\right.$ (flavonoid $-\mathrm{H}$ ) bipy] ${ }^{+}$complexes of three isomeric flavonoids (hesperidin, neohesperidin, and

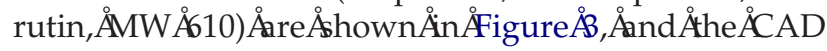
mass spectra of the other flavonoid complexes are

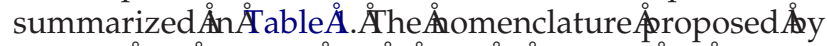

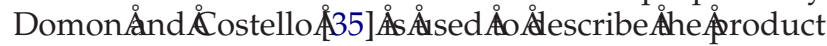
ions. Energy-variable CAD curves were acquired for all of the complexes and used to determine the dissociation threshold energies, which were defined as the CAD energies that cause conversion of $10 \%$ of the parent ions to fragment ions. To demonstrate the excellent reproducibility of such an approach, the energy-variable CAD curves of the $\left[\mathrm{Co}^{\mathrm{II}}\right.$ (neohesperidin $-\mathrm{H}$ ) bipy ${ }^{+}$

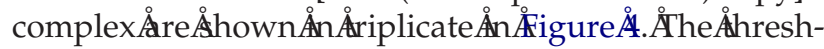
old energies of all the metal complexes (including the ones with different metals and/or auxiliary ligands) are

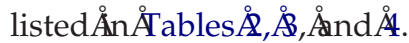



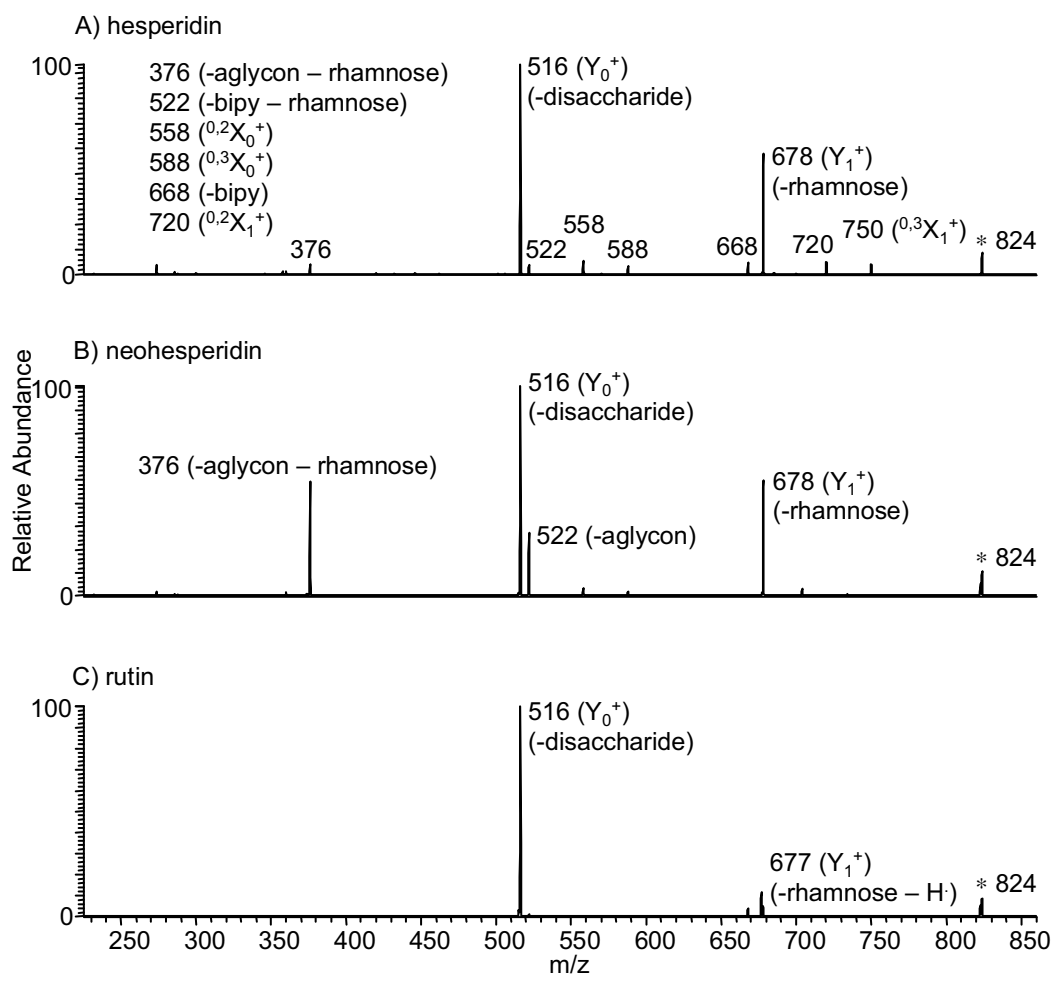

Figure 3. Representative CAD mass spectra of the $\left[\mathrm{Co}^{\mathrm{II}}(\mathrm{L}-\mathrm{H}) \text { bipy }\right]^{+}$complexes of hesperidin, neohesperidin and rutin. The parent complex ions are labeled with asterisks.

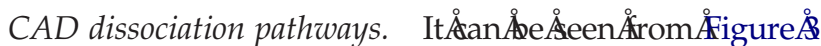
and Table 1 that the flavonoid diglycoside isomers are distinguishable based on the unique fragmentation patterns of the metal complexes. There are four prevalent fragmentation pathways of the metal complexes: loss of a single sugar residue (rhamnose or glucose), elimination of the entire disaccharide portion, loss of the aglycon moiety, or elimination of both the aglycon and rhamnose moieties. The dominant dissociation pathway of each flavonoid diglycoside/transition metal complex is the loss of the disaccharide portion, which results in a metal complex containing the aglycon and auxiliary ligand. The second most pronounced dissociation route is the loss of the rhamnose residue, resulting in the $\mathrm{Y}_{1}{ }^{+}$[35] Åon. Åhe Ånetal complexes that specifically contain neohesperidoside-type flavanones dissociate uniquely by the loss of the aglycon moiety, resulting in product ions in which the metal is bound to the disaccharide and the auxiliary ligand. Elimination of both the aglycon and rhamnose portions is consistently observed only for the metal complexes containing neohesperidoside-type flavonoids. The metal complexes of the O- bonded monoglycosides almost exclusively lose their sugar residues (glucose or rhamnose) upon $\mathrm{CAD}$, while the metal complexes of the $\mathrm{C}$ - bonded monoglycosides tend to undergo extensive cross-ring cleavages along with dehydration. The measurement of threshold energies offers information about the relative energetics of the dissociation behavior of the flavonoid complexes.

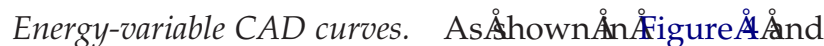

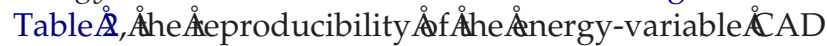
curves and threshold energies is satisfactory for our current study. For example, the relative standard deviation (RSD) of the threshold energies is only $3.8 \%$ for the worst case (the vitexin complex, $24.0 \pm 0.9 \%$ ). In most cases, the RSDs are quite low, typically less than $1 \%$ (i.e., on the order of $0.2 \%$ out of $25 \%$ threshold energy). Thus, the differences in threshold energies, though small in absolute values in some cases, are real and merit interpretation.

Threshold energies. Flavonoid diglycosides. The threshold dissociation energies of the $\left[\mathrm{Co}^{\mathrm{II}}(\mathrm{L}-\mathrm{H}) \text { bipy }\right]^{+}$ complexes were measured for five series of isomeric flavonoid diglycosides. In general, there are notable differences in the threshold energies measured for dissociation of the complexes containing different isomeric flavonoids, with consistent trends that reflect the type of disaccharide (rutinose versus neohesperidose), the type of aglycon (flavone or flavanone), and the type of saccharide linkage (O- bonded versus C- bonded). For example, hesperidin and neohesperidin are two flavanone glycosides that have the same aglycon (hesperetin) but different disaccharides. Hesperidin is a rutinoside flavanone with a 1-6 inter-saccharide linkage, whereas neohesperidin has a 1-2 linkage which makes it a neohesperidoside-type flavanone. This small difference in structure causes significant differences in the dissociation threshold energies of 


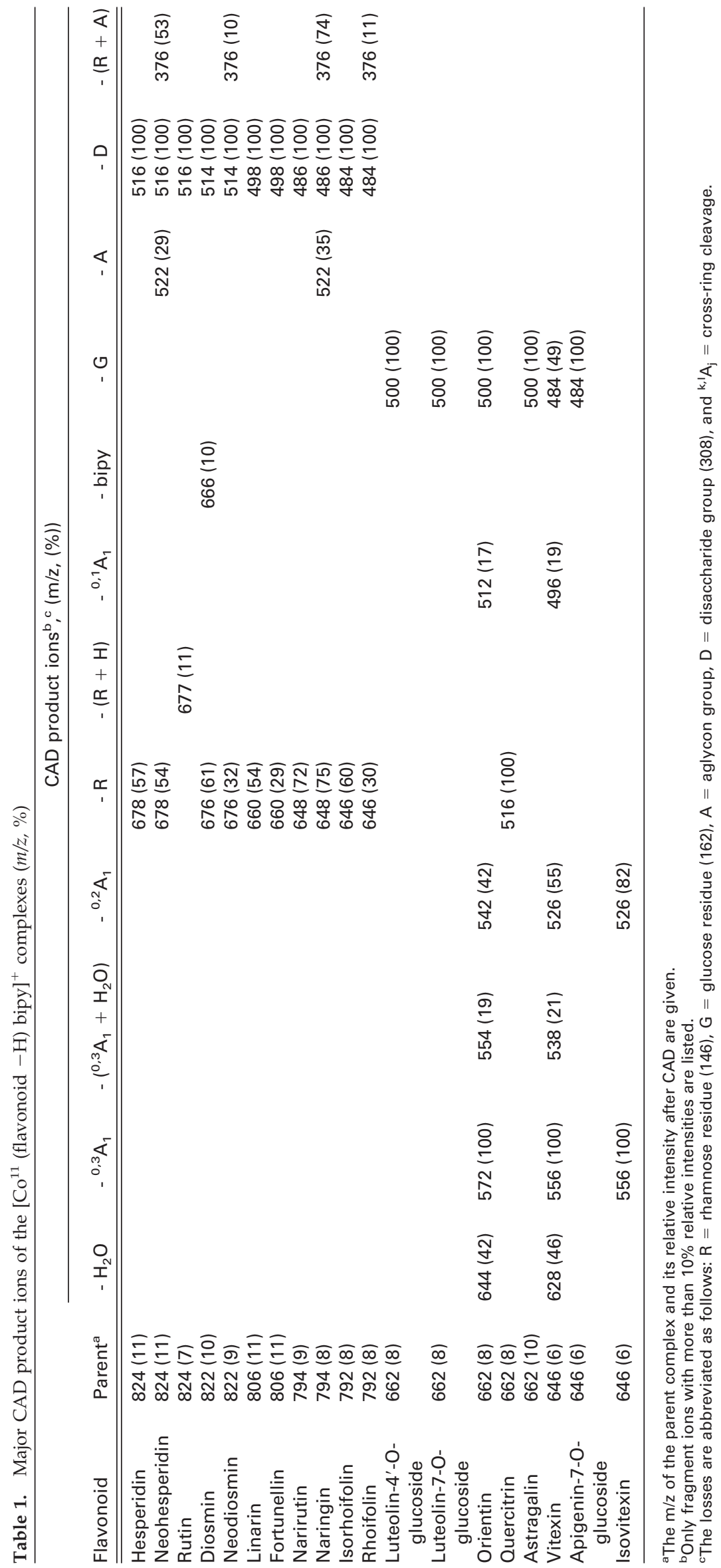




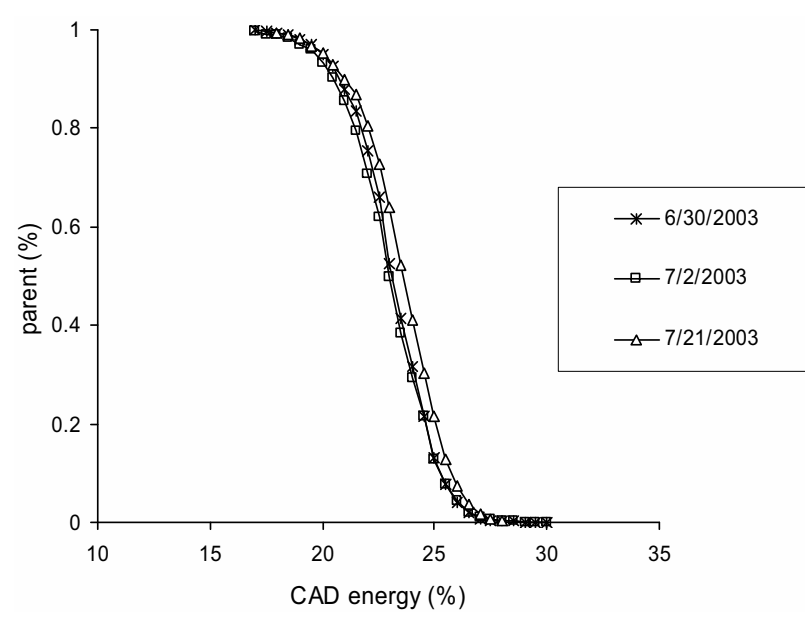

Figure 4. Energy-variable CAD curves of the $\left[\mathrm{Co}^{\mathrm{II}}\right.$ (neohesperidin $-\mathrm{H})$ bipy $]^{+}$complex in triplicate.

the metal complexes: the hesperidin complex requires $15 \%$ more CAD energy than the neohesperidin complex to dissociate For both of these isomers, the dominant fragmentation pathways of the metal complex [Co ${ }^{\text {II }}$ (flavonoid $-\mathrm{H}$ ) bipy $^{+}$is the loss of the disaccharide moiety, resulting in

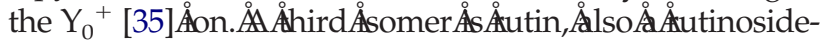
diglycoside like hesperidin but with the glycosylation site at the 3-position rather than the 7- position. The metal complex of rutin has the lowest threshold among the three

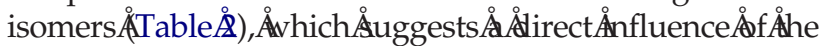
glycosylation site on the lability of the glycoside bond.

The metal complexes of the second flavanone isomeric pair, narirutin and naringin, give a similar trend in the dissociation energies to that observed for the
Table 3. Threshold dissociation energies (\%) of the $\left[\mathrm{M}^{\|}\right.$ (flavonoid $-\mathrm{H}$ ) bipy] ${ }^{+}$complexes

\begin{tabular}{lccc}
\hline Flavonoid & Co & $\mathrm{Ni}$ & $\mathrm{Cu}$ \\
\hline \hline rhoifolin & $24.3 \pm 0.3$ & $25.2 \pm 0.7$ & $25.9 \pm 0.6$ \\
$\begin{array}{c}\text { luteolin-4'-O- } \\
\text { glucoside }\end{array}$ & $26.1 \pm 0.2$ & $25.44 \pm 0.01$ & $26.11 \pm 0.04$ \\
$\begin{array}{c}\text { luteolin-7-O- } \\
\text { glucoside }\end{array}$ & $25.7 \pm 0.2$ & $25.1 \pm 0.3$ & $25.7 \pm 0.3$ \\
$\begin{array}{c}\text { apigenin-7-O- } \\
\text { glucoside }\end{array}$ & $26.0 \pm 0.5$ & $25.3 \pm 0.1$ & $25.78 \pm 0.01$ \\
\hline
\end{tabular}

hesperidin and neohesperidin complexes. The metal complex of the rutinoside isomer (narirutin) has a higher threshold energy than the complex of naringin, the neohesperidoside isomer. Again the dominant dissociation pathway is the loss of the disaccharide portion via cleavage across the glycosydic bond.

Similar to the two flavanone pairs described above, the metal complexes of each of the three flavone isomeric pairs show the same trend in the dissociation thresholds of the metal complexes: each rutinoside flavonoid complex has a greater threshold energy than

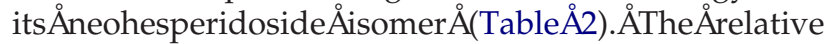
difference in the dissociation thresholds of the metal complexes of the isomeric flavones is about $6 \%$ (linarin versus fortunellin and isorhoifolin versus rhoifolin) or $7 \%$ (diosmin versus neodiosmin), which is about half the relative difference for a flavanone isomeric pair: $16 \%$ (hesperidin versus neohesperidin, and narirutin versus naringin). The larger threshold energy difference between the flavanones is presumed to be related to their single bond character between $\mathrm{C} 2$ and $\mathrm{C} 3$ on the aglycon moiety. The single bond allows free rotation of

Table 2. Threshold dissociation energies of the $\left[\mathrm{Co}^{\mathrm{II}}\right.$ (flavonoid $-\mathrm{H}$ ) bipy ${ }^{+}$complexes

\begin{tabular}{|c|c|c|c|c|c|c|}
\hline Flavonoid & MW & $\begin{array}{c}\text { Type of } \\
\text { saccharide }\end{array}$ & $\begin{array}{l}\text { Glycosylation } \\
\text { position }\end{array}$ & $\begin{array}{l}\text { Type of } \\
\text { aglycon }\end{array}$ & $m / z$ & $\begin{array}{l}\text { Threshold } \\
\text { energy }(\%)^{a}\end{array}$ \\
\hline Hesperidin & 610 & rutinose & $7-0$ & flavanone & 824 & $24.0 \pm 0.4$ \\
\hline Neohesperidin & 610 & neohesperidose & $7-0$ & flavanone & 824 & $20.8 \pm 0.2$ \\
\hline Rutin & 610 & rutinose & $3-0$ & flavonol & 824 & $18.3 \pm 0.4$ \\
\hline Diosmin & 608 & rutinose & $7-0$ & flavone & 822 & $26.3 \pm 0.2$ \\
\hline Neodiosmin & 608 & neohesperidose & $7-0$ & flavone & 822 & $24.6 \pm 0.1$ \\
\hline Linarin & 592 & rutinose & $7-0$ & flavone & 806 & $25.9 \pm 0.2$ \\
\hline Fortunellin & 592 & neohesperidose & $7-0$ & flavone & 806 & $24.5 \pm 0.5$ \\
\hline Narirutin & 580 & rutinose & $7-0$ & flavanone & 794 & $24.3 \pm 0.5$ \\
\hline Naringin & 580 & neohesperidose & $7-0$ & flavanone & 794 & $20.9 \pm 0.3$ \\
\hline Naringenin & 272 & & & flavanone & 486 & $32.8 \pm 0.3(59.6)^{b}$ \\
\hline Isorhoifolin & 578 & rutinose & $7-0$ & flavone & 792 & $25.8 \pm 0.1$ \\
\hline Rhoifolin & 578 & neohesperidose & $7-0$ & flavone & 792 & $24.3 \pm 0.3$ \\
\hline Luteolin-4'-O-glucoside & 448 & glucose & $4^{\prime}-0$ & flavone & 662 & $26.1 \pm 0.2$ \\
\hline Luteolin-7-O-glucoside & 448 & glucose & $7-0$ & flavone & 662 & $25.7 \pm 0.2$ \\
\hline Orientin & 448 & glucose & $8-C$ & flavone & 662 & $23.6 \pm 0.2$ \\
\hline Quercitrin & 448 & rhamnose & $3-0$ & flavonol & 662 & $19.2 \pm 0.1$ \\
\hline Astragalin & 448 & glucose & $3-0$ & flavonol & 662 & $11.8 \pm 0.2$ \\
\hline Vitexin & 432 & glucose & 8-C & flavone & 646 & $24.0 \pm 0.9$ \\
\hline Apigenin-7-O-glucoside & 432 & glucose & $7-0$ & flavone & 646 & $26.0 \pm 0.5$ \\
\hline Isovitexin & 432 & glucose & $6-\mathrm{C}$ & flavone & 646 & $21.6 \pm 0.4$ \\
\hline
\end{tabular}

aThe threshold energy is expressed as a percentage of $5 \mathrm{~V}_{0-\mathrm{p}}$.

bThe threshold corrected for degrees of freedom is listed in parenthesis. 
the $\mathrm{B}$ ring relative to the plane defined by the $\mathrm{A}$ and $\mathrm{C}$ rings, which may in turn result in conformations that allow more or less favorable interactions between the aglycon and the disaccharide depending on its identity (with a 1,2- or 1,6- inter-saccharide linkage). Direct evidence of such changes arises from molecular modeling, which is discussed later in section 3.2. Moreover, the thresholds of the complexes containing flavones are consistently greater than the thresholds of the analogous complexes containing flavanones, thus confirming the significant impact of a double bond versus a single bond between the $\mathrm{C} 2$ and $\mathrm{C} 3$ positions.

Metal complexes of flavonoid aglycons have much higher threshold energies than the complexes containing the corresponding flavonoid glycosides. For example, the metal complex of naringenin (the aglycon of narirutin and naringin) has a threshold energy of 32.8\%

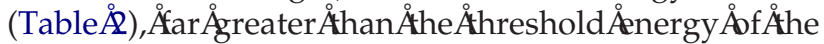
naringin or narirutin complex, which is not surprising because of the higher stability of the aglycon through conjugation and the lack of any labile bonds.

Flavonoid monoglycosides. Energy-resolved CAD experiments involving two flavonoid monoglycoside isomeric Åreries(Figure $\AA$ ) Åvere Åndertaken effects of the glycosylation position (3 versus 7 versus $4^{\prime}$ versus 6 versus 8 ) and linkage type (C- bonded versus $\mathrm{O}$ - bonded) on the threshold energies of the $\left[\mathrm{Co}^{\mathrm{II}}(\mathrm{L}-\right.$ $\mathrm{H})$ bipy] ${ }^{+}$complexes. Luteolin-4'-O-glucoside, luteolin7-O-glucoside, and orientin (luteolin-8-C-glucoside) are three luteolin glucosides that are glycosylated at the 4'-O-, 7-O-, and 8-C-positions, respectively, and quercitrin and astragalin (kaempferol-3-O-glucoside) are two 3-O- bonded isomers. The luteolin-4'-O-glucoside complex has the highest threshold energy among the isomeric series, and the two 3-O-glycosides have the lowest threshold energies.

The second isomeric series include apigenin-7-Oglucoside, vitexin, and isovitexin, which are 7-O-, 8-C-, and 6-C- bonded glucosides of the same aglycon, apigenin. The complex of apigenin-7-O-glucoside, the only $\mathrm{O}$ - bonded one, has a greater threshold energy than either of the C- bonded glucoside complexes, and the 6-C-glucoside (isovitexin) has a lower threshold than that of the corresponding 8-C-glucoside isomer (vitexin) (Table \&).

In general, the threshold energies follow these three trends: O- bonded > C- bonded; 4'-O $>7-\mathrm{O}>3-\mathrm{O}-$ bonded; and 8-C $>6-\mathrm{C}$ - bonded. For either an Obonded or C- bonded glycoside, if the glycosylation position is farther away (4'- or 7- or 8-) from the metal complexation sites (4- and 5- in general), the complex requires a higher $\mathrm{CAD}$ energy. If the glycosylation position is much closer (3- or 6-) to the complexation sites, the complex requires a lower CAD energy.

Effects of transition metals and auxiliary ligands. In addition to the $\left[\mathrm{Co}^{\mathrm{II}}(\mathrm{L}-\mathrm{H}) \text { bipy }\right]^{+}$complexes, the effects of different transition metals (cobalt, nickel, and copper) and auxiliary ligands (bipy, phen, dmphen, and dpphen) on the threshold energies of the metal complexes were also studied for several of the flavonoids. These results ̊re

Transition metals. When nickel or copper is used for metal complexation instead of cobalt, some of the minor fragmentation pathways of the flavonoid complexes are quenched. For example, the minor loss of the aglycon portion, a pathway observed for the cobalt complexes of luteolin-7-O-glucoside or apigenin-7-O-glucoside, is not observed upon CAD of the nickel or copper complexes. The loss of both the aglycon and rhamnose moieties, which is apparent for the cobalt complex of rhoifolin, is not observed for the nickel or copper complexes. The cobalt, nickel and copper complexes of rhoifolin, luteolin-4'-O-glucoside, luteolin-7-O-glucoside, and apigenin-7-O-glucoside, with 2,2'-bipyridine as the auxiliary ligand, have very similar threshold energies, respectively $\AA$ (TableÅ3),Åsuggesting Åthat ÅtheÅidentity Åof Åthe divalent transition metal does not have a notable impact on the stability of the complexes.

Auxiliary ligands. The transition metal/flavonoid complexes show substantial differences in the threshold

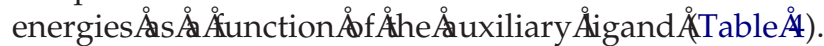
The same major fragmentation pathways occur for the complexes containing different auxiliary ligands, and again the dominant route at the threshold level is the loss of the disaccharide group. Both the experimentally determined threshold energies and the ones obtained

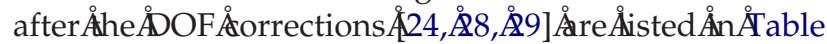
4,Åbearing Åin Åmind Åthat ÅtheÅsimpleÅDOFÅcorrection procedure does not account for variations in the initial internal or kinetic energies of the complexes. The corrected threshold energies for the complexes decrease from bipy to phen to dmphen or dpphen, with the most significant decrease for the complexes containing the dpphen auxiliary ligand. It is plausible that the large, most polarizable dpphen ligand donates more electron density to the metal ion, which thus alters the electron density distribution through the attached flavonoid. This shift in electron density could influence the energy required for loss of the disaccharide portion upon collisional activation of the complexes.

Table 4. Threshold dissociation energies (\%) of the [Coll (flavonoid $-\mathrm{H}$ ) auxiliary ligand $]^{+}$complexes $^{\mathrm{a}}$

\begin{tabular}{lccrr}
\hline Flavonoid & bipy & phen & dmphen & dpphen \\
\hline \hline Rhoifolin & $24.3 \pm 0.3$ & $24.4 \pm 0.4(23.8)$ & $25.1 \pm 0.2(23.0)$ & $25.9 \pm 0.3(20.8)$ \\
Luteolin-4'-O-glucoside & $26.1 \pm 0.2$ & $26.2 \pm 0.1(25.5)$ & $27.19 \pm 0.05(24.40)$ & $29.0 \pm 0.2(22.1)$ \\
\hline
\end{tabular}

aThe thresholds corrected for degrees of freedom are listed in parentheses. 


\section{Molecular Modeling}

Despite the proven analytical utility of the transition

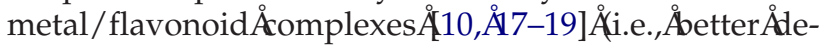
tection limits and differentiation of isomers based on CAD), the structures of the transition metal complexes of the flavonoids have not been modeled previously. Therefore, extensive molecular modeling was carried out on five model flavonoids (hesperidin, neohesperidin, rutin, diosmin, and neodiosmin), including both their cobalt complexes and deprotonated molecules, by $\mathrm{QM}$ and MM means. For the transition metal complexes, the models entail deprotonation of the $5-\mathrm{OH}$ moiety and chelation of the metal via the resulting deprotonated 5-O site and the adjacent carbonyl oxygen. This favorable metal coordination site has been confirmed previously via experiments involving an array of flavonoids that possessed or lacked the key

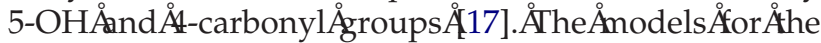
deprotonated flavonoids typically involve deprotonation of a hydroxyl group on the $\mathrm{B}$ ring, not the $5-\mathrm{OH}$ on the A ring. The most favorable sites of deprotonation of flavonoids in the gas phase have also been elucidated in

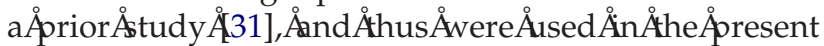
study for the molecular modeling.

Coordination geometries and structural parameters. By a plane-angle scanning study at the B3LYP/6-31G* level, two stable coordination geometries were identified for the [Co ${ }^{\text {II }}$ (flavonoid - H) 2,2'-bipyridine] complexes containing the model compound 5,7-dihydroxy-4-

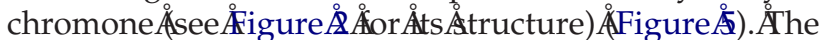

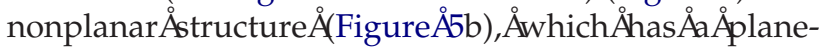
angle of $61.97^{\circ}$, is approximately $5.5 \mathrm{kcal} / \mathrm{mol}$ more

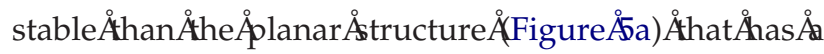
plane-angle of $179.49^{\circ}$. Therefore, only the nonplanar geometry was considered for the successive molecular modeling Åstudies.ÅAlso ̊̊shown Åin $̊$ Figure Å5 ÅareÅthe structural parameters for the cobalt/oxygen and co-

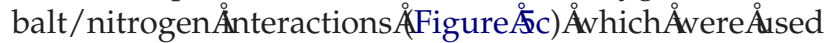
for force field parameterization.

Force field parameters. The missing $\mathrm{MM}$ force field parameters (parameters involving cobalt) were developed as described briefly in the experimental section and optimized to reproduce both the QM structure and the vibrational frequencies. For the model complex shownÅin $A ̊ F i g u r e \AA ̊ 5 b$, Åthe Åroot-mean-squareÅdeviation (RMSD) of atomic displacement between the QM and MM structures is $0.14 \AA$ and the RMSD of twelve vibrational frequencies involving cobalt is $37.93 \mathrm{~cm}^{-1}$.

Conformations. Ten genetic algorithm (GA) runs achieved similar conformations for each cobalt complex or deprotonated flavonoid, implying that the homebuilt GA program is robust and reproducible in conformation studies. MD simulations were applied to further relax the structures without any structural degrees of freedom frozen. The minimized structures of five cobalt complexes and five deprotonated flavonoids are shown in Âigures complexes that the disaccharide folds towards the metal ion which is chelated by oxygen atoms on the A and C rings, a feature most pronounced for the rutinoside flavonoids. In contrast, for the corresponding deprotonated flavonoids, the disaccharide portion tends to fold toward the deprotonation site on the B ring of the

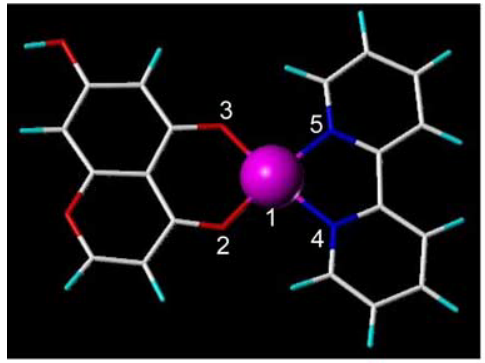

(a) plane-angle $=179.49^{\circ}$

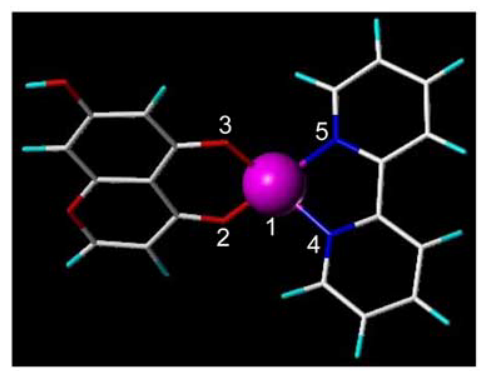

(b) plane-angle $=61.80^{\circ}$
(C) structural parameters

\begin{tabular}{|c|c|c|}
\hline $\begin{array}{l}\text { structural } \\
\text { parameter }\end{array}$ & $\begin{array}{l}\text { planar (plane- } \\
\left.\text { angle }=179.49^{\circ}\right)\end{array}$ & $\begin{array}{l}\text { non-planar } \\
\text { (plane-angle }= \\
61.97^{\circ} \text { ) }\end{array}$ \\
\hline \multicolumn{3}{|c|}{ bond length $(\AA)$} \\
\hline $1-2$ & 1.783 & 1.755 \\
\hline $1-3$ & 1.824 & 1.786 \\
\hline $1-4$ & 1.783 & 1.755 \\
\hline $1-5$ & 1.824 & 1.786 \\
\hline \multicolumn{3}{|c|}{ bond angle $\left({ }^{\circ}\right)$} \\
\hline $2-1-3$ & 93.57 & 101.11 \\
\hline $4-1-5$ & 93.57 & 101.10 \\
\hline $2-1-4$ & 88.50 & 116.17 \\
\hline $3-1-5$ & 84.46 & 111.56 \\
\hline \multicolumn{3}{|c|}{ torsional angle $\left({ }^{\circ}\right)$} \\
\hline $2-3-1-4$ & -174.0 & 125.2 \\
\hline $2-3-1-5$ & 179.8 & -121.2 \\
\hline $2-4-1-3$ & 174.0 & -116.7 \\
\hline $2-4-1-5$ & -179.8 & 123.6 \\
\hline
\end{tabular}

Figure 5. Stable coordination geometries and structural parameters of the $\left[\mathrm{Co}^{\mathrm{II}}\right.$ (5,7-dihydroxy-4chromone $-\mathrm{H}$ ) bipy ${ }^{+}$complex identified by the B3LYP $/ 6-31 G^{*}$ plane-angle scanning. 


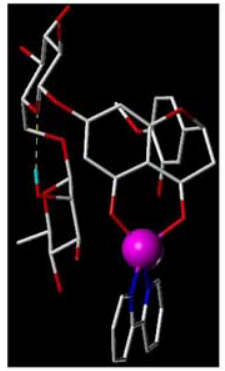

(a) hesperidin

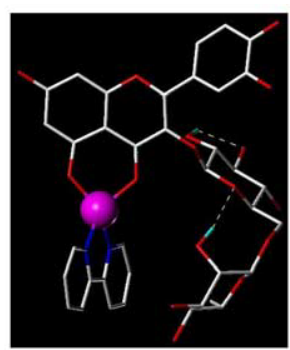

(b) rutin

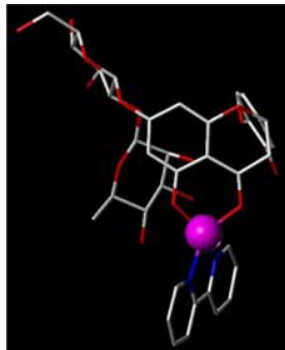

(c) neohesperidin

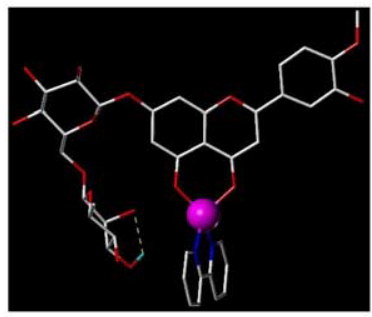

(d) diosmin

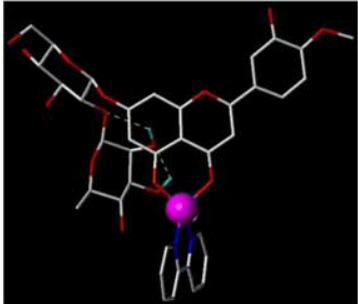

(e) neodiosmin

Figure 6. The last snapshots of the $\left[\mathrm{Co}^{\mathrm{II}}(\mathrm{L}-\mathrm{H}) \text { bipy }\right]^{+}$complexes via MD simulations. Hydrogen bonds are shown as dashed yellow lines and cobalt is shown as a magenta ball.

aglycon, and the disaccharide and aglycon moieties are linked by hydrogen bond(s). The conformational changes upon transition metal complexation may contribute to some of the altered fragmentation pathways of the metal complexes, which thus allow enhanced isomer differentiation.

Point charges and helium accessible surface areas. Based on the fact that it is a formidable endeavor to map the transition states for transition metal containing systems by using ab initio approaches, here we propose a simple empirical hypothesis to qualitatively interpret the threshold CAD data of the $\left[\mathrm{Co}^{\mathrm{II}}\right.$ (flavonoid $-\mathrm{H}$ ) $2,2^{\prime}$-bipyridine $]^{+}$complexes. The hypothesis is built on the concept that in a CAD process the apparent threshold or activation energy of a fragmentation reaction is dependent on the strength of the cleaved bond and the collision cross section (i.e., the probability of a collision

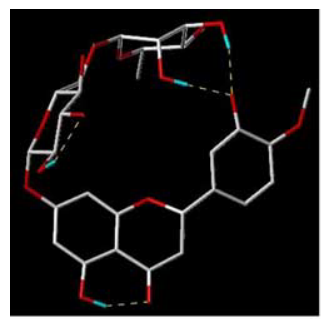

(a) hesperidin

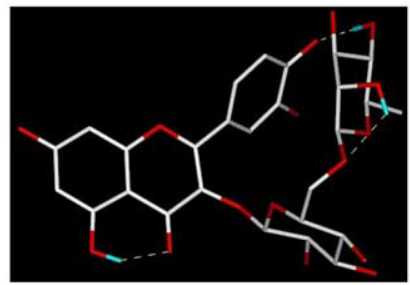

(b) rutin

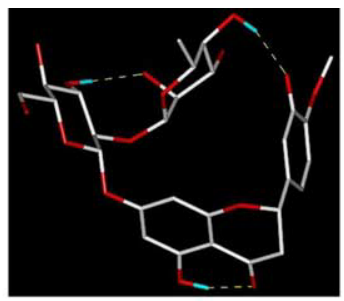

(c) neohesperidin

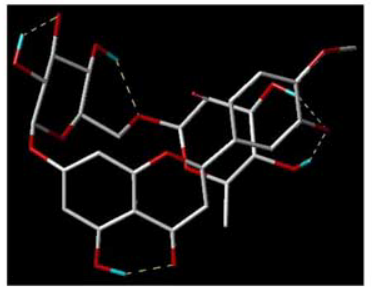

(d) diosmin

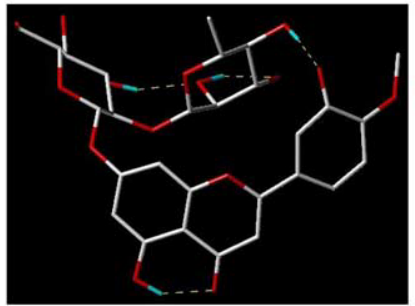

(e) neodiosmin

Figure 7. The last snapshots of deprotonated flavonoids obtained via MD simulation. Hydrogen bonds are shown as dashed yellow lines. 
based on the sizes of the collision gas and analyte ion). The bond strength is a function of bond length and the product of point charges of the two bonded atoms. In this study, only the $\mathrm{C}-\mathrm{O}$ bond linking the aglycon portion and disaccharide portion of a complex was considered because loss of the disaccharide moiety is the most dominant fragmentation pathway. The $\mathrm{C}-\mathrm{O}$ bond length does not vary to any significant extent for the isomeric flavonoids, thus this factor has a marginal impact for different flavonoids. However, the point charges vary considerably for the oxygen and carbon atoms of the key $\mathrm{C}-\mathrm{O}$ bond that is cleaved. As the product of the two point charges decreases, the bond strength decreases and therefore the activation energy of the dissociation reaction is expected to diminish. It should be pointed out that the point charges of the $\mathrm{C}$ and $\mathrm{O}$ atoms in the $\mathrm{C}-\mathrm{O}$ bond may differ between the ground state and transition state(s), respectively, for a specified complex. Yet it is generally true that the stronger the bond in the ground state, the more difficult it is to break for structurally similar species (isomeric complexes in this case) when similar transition state(s) are involved. Though not quantitatively accurate, the use of point charges in the ground states should reflect the bond strengths qualitatively. The collision crosssection areas can be estimated by the helium (collision gas) accessible surface areas of the complexes. As the calculated areas increase, the probability of collision increases at a specific collision gas pressure and time (identical for isomeric flavonoid complexes). Although the nature of the collisional activation process involves a mixture of both activating and deactivating collisions, it should be reasonable to assume that the percentage of activating collisions (number of activating collisions/ number of total collisions) for isomeric complexes is relatively constant as long as the experimental parameters are held constant (i.e., same helium pressure, same activation time, same $\mathrm{q}_{\mathrm{z}}$ value, same $m / z$ value of precursor ion). Therefore, the larger the surface area of a complex, the more activating collisions it should undergo. In addition, isomeric complexes have the same number of degrees of freedom and thus they should have similar rates of energy deposition per collision. It is thus a plausible assumption that complexes with larger helium accessible surface areas have concomitant lower apparent dissociation thresholds than their isomers with smaller surface areas when the other conditions are the same.

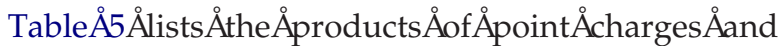
helium accessible surface areas of the cobalt complexes. It can be seen from this table that the accessible surface area of the hesperidin complex is slightly lower than that of the neohesperidon complex, implying a relatively (but probably not significantly) lower cross section, but the point charge products are quite different, with the one for the $\mathrm{C}-\mathrm{O}$ bond of the hesperidin complex substantially greater and implying a stronger bond. As a result, the threshold energy of the hesperidin complex is predicted to be higher than that of the
Table 5. The point charges and helium accessible surface areas of the oxygen and carbon atoms involved in bond-breaking of the cobalt complexes of flavonoids

\begin{tabular}{|c|c|c|c|c|}
\hline \multirow[b]{2}{*}{ Flavonoid } & \multicolumn{3}{|c|}{ Point charge $^{a, b}$} & \multirow{2}{*}{$\begin{array}{c}\text { Helium } \\
\text { accessible } \\
\text { surface area } \\
\left(\AA^{2}\right)\end{array}$} \\
\hline & $q_{o}$ & $\mathrm{q}_{\mathrm{c}}$ & $-q_{o} \times q_{c}$ & \\
\hline hesperidin & -0.261 & 0.506 & 0.132 & 894.3 \\
\hline neohesperidin & -0.216 & 0.427 & 0.092 & 919.3 \\
\hline rutin & -0.220 & 0.506 & 0.112 & 1032 \\
\hline diosmin & -0.270 & 0.506 & 0.137 & 1029 \\
\hline neodiosmin & -0.225 & 0.427 & 0.096 & 1102 \\
\hline
\end{tabular}

${ }^{a}$ Only the oxygen and carbon atoms involved in breaking the aglycondisaccharide bond are considered.

${ }^{b}$ All the charges are spliced HF/6-31G* RESP charges.

neohesperidin complex, and this prediction is borne out

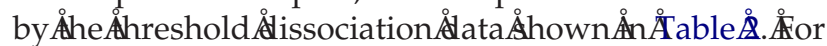
the diosmin and neodiosmin complexes, the product of the point charges is greater for the diosmin complex, suggesting a stronger bond, and the corresponding helium accessible surface area is lower than that of the neodiosmin complex, indicating a smaller crosssectional area for collisional activation. These two factors are consistent with the greater threshold energy of the diosmin complex relative to the neodiosmin complex. The rutin complex is different from the above four since it has a different glycosylation site (3- instead of 7-). The medium bond strength and the largest helium accessible surface area make the rutin complex the easiest to dissociate among its isomers, which seems to indicate that the significantly higher surface area of the rutin complex plays a more important role than its product of point charges in its dissociation. Of course, it can not be excluded that the distinct 3-glycosylation of rutin makes its complex behave quite differently from the isomeric hesperidin and neohesperidin complexes (for example, it is known that 3-glycosylated flavonoids undergo acid hydrolysis more rapidly than the isomeric

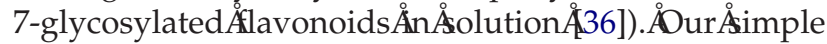
hypothesis has its own limitations and it is most applicable to structurally very similar species, such as cobalt/bipy complexes of rutinosides and their isomeric neohesperidosides.

The rationalization of the CAD threshold data for these five isomers can be extended to the other isomers as well. Based on our modeling studies, it is likely that the flavanones in general form more compact complexes than their flavone counterparts because the flavanones have a more flexible single bond rather than a double bond between C2 and C3. The smaller collisional cross sections of the compact flavanone complexes should result in corresponding lower collision probabilities. Likewise, the point charges (and corresponding bond energies) for the aglycon-disaccharide bond of the rutinosides are greater than those of the corresponding neohesperidosides, implying greater dissociation energies for the former. 


\section{Conclusions}

Energy-variable collisionally activated dissociation has been used in this work to compare the threshold dissociation energies of the metal complexes of seven isomeric series of flavonoid glycosides and to correlate the threshold energies with structural features. For the metal complexes of the flavonoid diglycosides, rutinosides consistently have higher CAD threshold energies than their neohesperidoside counterparts, and the differences in the threshold energies are larger for the flavanones than the corresponding flavones. For the metal complexes of the monoglycosides, C- bonded flavonoids tend to have lower threshold energies than the $\mathrm{O}$ - bonded isomers. The site of glycosylation also has a significant effect on the dissociation threshold energies of both the mono- and diglycosides with the 3-glycosylated flavonoids having the lowest threshold energies among the isomers. The influence of the type of transition metal and the auxiliary ligand on the dissociation thresholds has also been investigated. There is no significant difference in the threshold energies of the flavonoid complexes with the three tested transition metals (cobalt, nickel, and copper).

Two stable coordination geometries have been identified for the metal complexes, and the complexes are nonplanar based on the calculated energies. After force field parameterization, conformational searches, and MD simulations, the most favorable conformations of five representative cobalt complexes and five corresponding deprotonated flavonoids have been obtained. The combination of point charges and helium accessible surface areas gives reasonable explanations for the trends in the CAD threshold energies. The dissociation threshold energies and fragmentation patterns could be used in combination to differentiate known flavonoid isomers and to potentially unravel the structures of new flavonoids.

\section{Acknowledgments}

This work was supported by the Welch Foundation (F-1155) and the National Institutes of Health (R01-GM63512). The authors acknowledge NCSA for providing the supercomputing time (account no. MCB000017N; PI: JW).

\section{References}

1. Bohm, B. A. Introduction to Flavonoids; Harwood Academic Publishers: Amsterdam, 1998; p 365

2. Middleton, E., Jr.; Kandaswami, C. In The Flavonoids: Advances in Research since 1986; Harborne, J. B., Ed.; Chapman and Hall: London, 1994; p 619.

3. Kaur, C; Kapoor, H. C. Antioxidants in fruits and vegetablesthe millennium's health. Int. J. Food Sci. Technol. 2001, 36, 703-725.

4. Justesen, U. Negative atmospheric pressure chemical ionization low-energy collision activation mass spectrometry for the characterization of flavonoids in extracts of fresh herbs. J.Chromatogr. A 2000, 902, 369-379.
5. Justesen, U. Collision-induced fragmentation of deprotonated methoyxlated flavonoids, obtained by electrospray ionization mass spectrometry. J. Mass Spectrom. 2001, 36, 169-178.

6. Fabre, N.; Rustan, I.; de Hoffmann, E.; Quetin-Leclercq, J. Determination of flavone, flavonol, and flavanone aglycones by negative ion liquid chromatography electrospray ion trap mass spectrometry. J. Am. Soc. Mass Spectrom. 2001, 12, 707715.

7. Cuyckens, F.; Rozenberg, R.; de Hoffmann, E.; Claeys, M. Structural characterization of flavonoid O-diglycosides by positive and negative nano-electrospray ionization ion trap mass spectrometry. J. Mass Spectrom. 2001, 36, 1203-1210.

8. Hughes, R. J.; Croley, T. R.; Metcalfe, C. D.; March R. E. A tandem mass spectrometric study of selected characteristic flavonoids. Int. J. Mass Spectrom. 2001, 210/211, 371-385.

9. Hvattum, E.; Ekeberg, D. Study of the collision-induced radical cleavage of flavonoid glycosides using negative electrospray ionization tandem quadrupole mass spectrometry. $J$. Mass Spectrom. 2003, 38, 43-49.

10. Zhang, J.; Brodbelt, J. S. Structural characterization and isomer differentiation of chalcones by electrospray ionization tandem mass spectrometry. J. Mass Spectrom. 2003, 38, 555-572.

11. Zhang, J.; Satterfield, M. B.; Brodbelt, J. S.; Britz, S. J.; Clevidence, B.; Novotny, J. A. Structural characterization and improved detection of kale flavonoids by electrospray ionization mass spectrometry. Anal. Chem. 2003, 75, 6401-6407.

12. Ma, Y. L.; Li, Q.; Van den Heuvel, H.; Claeys, M. Characterization of flavone and flavonol aglycones by collision-induced dissociation tandem mass spectrometry. Rapid Commun. Mass Spectrom. 1997, 11, 1357-1364.

13. Ma, Y. L.; Vedernikova, I.; Van den Heuvel, H.; Claeys, M. Internal glucose residue loss in protonated O-diglycosyl flavonoids upon low-energy collision-induced dissociation. J. Am. Soc. Mass Spectrom. 2000, 11, 136-144.

14. Ma, Y. L.; Cuyckens, F.; Van den Heuvel, H.; Claeys, M. Mass spectrometric methods for the characterization and differentiation of isomeric O-diglycosyl flavonoids. Phytochem. Anal. 2001, 12, 159-165.

15. Cuyckens, F.; Shahat, A. A.; Pieters, L.; Claeys, M. Direct stereochemical assignment of hexose and pentose residues in flavonoid O-glycosides by fast atom bombardment and electrospray ionization mass spectrometry. J. Mass Spectrom. 2002, 37, 1272-1279.

16. Franski, R.; Matlawska, I.; Bylka, W.; Sikorska, M.; Fiedorow, P.; Stobiecki, M. Differentiation of interglycosidic linkages in permethylated flavonoids from linked-scan mass spectra (B/ E). J. Agric. Food Chem. 2002, 50, 976-982.

17. Satterfield, M.; Brodbelt, J. Enhanced detection of flavonoids by metal complexation and electrospray ionization mass spectrometry. Anal. Chem. 2000, 72, 5898-5906.

18. Satterfield, M.; Brodbelt, J. S. Structural characterization of flavonoid glycosides by collisionally activated dissociation of metal complexes. J. Am. Soc. Mass Spectrom. 2001, 12, 537-549.

19. Pikulski, M.; Brodbelt, J. S. Differentiation of flavonoid glycoside isomers by using metal complexation and electrospray ionization mass spectrometry. J. Am. Soc. Mass Spectrom. 2003, 14, 1437-1453.

20. Harborne, J. B. Nature, distribution, and function of plant flavonoids. Prog. Clin. Biol. Res. 1986, 213, 15-24.

21. Hart, K. J.; McLuckey, S. A. Relative dissociation energy measurements using ion trap collisional activation. J. Am. Soc. Mass Spectrom. 1994, 5, 250-259.

22. Colorado, A.; Brodbelt, J. S. An empirical approach to estimation of critical energies by using a quadrupole ion trap. J. Am. Soc. Mass Spectrom. 1996, 7, 1116-1125.

23. Satterfield, M. B.; Brodbelt, J. S. Relative binding energies of gas-phase pyridyl ligand/metal complexes by energy-variable 
collisionally activated dissociation in a quadrupole ion trap. Inorg. Chem. 2001, 40, 5393-5400.

24. Jellen, E. E.; Chappell, A. M.; Ryzhov, V. Effect of size of noncovalent complexes on their stability during collisioninduced dissociation. Rapid Commun. Mass Spectrom. 2002, 16, 1799-1804.

25. Hayes, L. A.; Chappell, A. M.; Jellen, E. E.; Ryzhov, V. Binding of metalloporphyrins to model nitrogen bases: Collision-induced dissociation and ion-molecule reaction studies. Int. J. Mass Spectrom. 2003, 227, 111-120.

26. David, W. M.; Brodbelt, J. S. Threshold dissociation energies of protonated amine/polyether complexes in a quadrupole ion trap. J. Am. Soc. Mass Spectrom. 2003, 14, 383-392.

27. Crowe, M. C.; Brodbelt, J. S. Evaluation of noncovalent interactions between peptides and polyether compounds via energy-variable collisionally activated dissociation. J. Am. Soc. Mass Spectrom. 2003, 14, 1148-1157.

28. Dongre, A. R.; Dones, J. L.; Somogyi, A.; Wysocki, V. H. Influence of peptide composition, gas-phase basicity, and chemical modification on fragmentation efficiency: Evidence for the mobile proton model. J. Am. Chem. Soc. 1996, 118, 8365-8374.

29. Vachet, R. W.; Glish, G. L. New method to study the effects of peptide sequence on the dissociation energies of peptide ions. J. Am. Soc. Mass Spectrom. 1998, 9, 175-177.

30. Frisch, M. J.; Trucks, G. W.; Schlegel, H. B.; Scuseria, G. E.; Robb, M. A.; Cheeseman, J. R.; Montgomery, J. A., Jr.; Vreven, T.; Kudin, K. N.; Burant, J. C.; Millam, J. M.; Iyengar, S. S.; Tomasi, J.; Barone, V.; Mennucci, B.; Cossi, M.; Scalmani, G.; Rega, N.; Petersson, G. A.; Nakatsuji, H.; Hada, M.; Ehara, M.; Toyota, K.; Fukuda, R.; Hasegawa, J.; Ishida, M.; Nakajima, T.; Honda, Y.; Kitao, O.; Nakai, H.; Klene, M.; Li, X.; Knox, J. E.; Hratchian, H. P.; Cross, J. B.; Adamo, C.; Jaramillo, J.; Gomperts, R.; Stratmann, R. E.; Yazyev, O.; Austin, A. J.; Cammi, R.;
Pomelli, C.; Ochterski, J. W.; Ayala, P. Y.; Morokuma, K.; Voth, G. A.; Salvador, P.; Dannenberg, J. J.; Zakrzewski, V. G.; Dapprich, S.; Daniels, A. D.; Strain, M. C.; Farkas, O.; Malick, D. K.; Rabuck, A. D.; Raghavachari, K.; Foresman, J. B.; Ortiz, J. V.; Cui, Q.; Baboul, A. G.; Clifford, S.; Cioslowski, J.; Stefanov, B. B.; Liu, G.; Liashenko, A.; Piskorz, P.; Komaromi, I.; Martin, R. L.; Fox, D. J.; Keith, T.; Al-Laham, M. A.; Peng, C. Y.; Nanayakkara, A.; Challacombe, M.; Gill, P. M. W.; Johnson, B.; Chen, W.; Wong, M. W.; Gonzalez, C.; Pople, J. A. Gaussian 03; Gaussian, Inc.: Pittsburgh, PA, 2003.

31. Zhang, J.; Brodbelt, J. S. Gas-phase hydrogen/deuterium exchange and conformations of deprotonated flavonoids and gas-phase acidities of flavonoids. J. Am. Chem. Soc. 2004, 126, 5906-5919.

32. Case, D. A., Darden, T. A., Cheatham, T. E. III, Simmerling, C. L., Wang, J., Duke,, R. E., Luo,, R., Merz, K. M., Wang, B., Pearlman, D. A., Crowley, M., Brozell, S., Tsui, V., Gohlke, H., Mongan, J., Hornak, V., Cui, G., Beroza, P., Schafmeister, C., Caldwell, J. W., Ross, W. S., Kollman, P. A. AMBER 8; University of California, San Francisco, 2004.

33. Wang, J.; Cieplak, P.; Kollman, P. A. How well does a restrained electrostatic potential (RESP) model perform in calculating conformational energies of organic and biological models? J. Comput. Chem. 2000, 21, 1049-1074.

34. Wang, J.; Wolf, R.; Caldwell, J.; Kollman, P.; Case, D. Development and testing of a general AMBER force field. J. Comput. Chem. 2004, 25, 1157-1174.

35. Domon, B.; Costello, C. E. A systematic nomenclature for carbohydrate fragmentations in FAB-MS/MS spectra of glycoconjugates. Glycoconjugate J. 1998, 5, 397-409.

36. Harborne, J. B. Plant polyphenols XIV. Characterization of flavonoid glycosides by acidic and enzymic hydrolysis. Phytochemistry 1965, 4, 107-120. 